\title{
Síndrome de Seckel, Dos Casos en una Familia Colombiana
}

\author{
HARRY PACHAJOA ${ }^{1}$, WILMAR SALDARRIAGA ${ }^{2}$, CAROLINA ISAZA $^{3}$ \\ 1. MD, Est PhD, Profesor y Jefe Departamento de Ciencias Básicas Medicas, Facultad de Ciencias de la Salud, \\ Universidad Icesi, Cali, Colombia. \\ 2. MD, MSc, Ginecólogo y Obstetra, Profesor Asistente, Facultad de Salud, Universidad del Valle, Cali, Colombia \\ 3. MD, MSc, Profesora Titular, Facultad de Salud, Universidad del Valle, Cali, Colombia.
}

\begin{abstract}
Seckel Syndrome. Report of Two Cases in a Colombian Family

Seckel syndrome is an infrequent autosomic recessive genetic disorder. It is characterized by short stature, mental retardation and a typical facies. Nearly 10 families have been reported with two or more affected members. This paper reports two sisters, daughters of non-related parents. The mother presented bilateral fissurate lip. The main traits of this syndrome are highlighted through a literature review.

(Key words: Short stature, Seckel syndrome, inheritance pattern, mycrocephalia).

Rev Chil Pediatr 2010; 81 (5): 432-436
\end{abstract}

\section{RESUMEN}

El síndrome de Seckel es una enfermedad genética de herencia autosómica recesiva de baja ocurrencia, caracterizado por la asociación de talla baja, retardo mental y facies especial, se han reportado cerca de 10 familias con dos o más miembros afectados. Nosotros reportamos dos hermanas hijas de padres no consanguíneas, la madre presenta labio fisurado bilateral. Se describen las características más importantes del síndrome y se hace una revisión de la literatura.

(Palabras clave: Enanismo, síndrome de Seckel, microcefalia, patrón de herencia).

Rev Chil Pediatr 2010; 81 (5): 432-436

\section{Introducción}

El síndrome de Seckel es de herencia autosómica recesiva ${ }^{1}$ (MIM 210600) y se caracteriza por la asociación de talla baja, retardo mental y facies dismórficas que incluyen microcefalia severa, frente prominente, ojos grandes, protrusión de la nariz, cara pequeña y micrognatia. Hasta el año 2002 se habían reportado cerca de 100 casos diagnosticados clínicamente ${ }^{2}$.

Se han reportado cerca de 10 familias don-

Trabajo recibido el 21 de enero de 2010, devuelto para corregir el 05 de mayo de 2010, segunda versión el 31 de mayo de 2010, tercera versión el 21 de junio de 2010, aceptado para publicación el 05 de agosto de 2010.

Correspondencia a:

Dr. Harry Pachajoa

E-mail: harrympl@yahoo.com 
de se reportan 2 o más miembros de la familia afectados (tabla 1$)^{3-9}$.

Se presentan 2 casos de síndrome de Seckel en una misma familia colombiana, siendo este el primer reporte de este tipo en población latinoamericana. Los pacientes son hijos de una madre con antecedente de labio y paladar fisurado bilateral, talla baja (1,50 mts), sin antecedentes de consaguinidad (figuras 1 y 2 ).

\section{Paciente 1}

Producto de segundo embarazo, con edad materna al nacimiento de 37 años a quien se le atendió parto hospitalario a las 37 semanas, al nacimiento peso de $1070 \mathrm{~g}$ $(\mathrm{p}<5)$, talla: $32 \mathrm{~cm}(\mathrm{p}<5)$ sexo femenino. En el momento de la consulta se encontró paciente de dos años con peso de 3500 $\mathrm{g}(\mathrm{p}<5)$, talla: $52 \mathrm{~cm}(\mathrm{p}<5)$, perímetro cefálico de $37 \mathrm{~cm}(p<5)$, distancia intercantal interna de $2 \mathrm{~cm}(\mathrm{p}<5)$, tamaño total de la mano de $4,8 \mathrm{~cm}(\mathrm{p}<5)$, al examen físico se encontró microcefalia, micrognatia, fisuras palpebrales inclinadas hacia abajo, nariz prominente, paladar ojival, clinodactilia bilateral y pie equino varo derecho (figuras 3 y 4), trae radiografía de huesos largos que evidenció retardo en la aparición de los núcleos epifisiarios y 11 costillas.

No fue realizado estudio de cariotipo.

\section{Paciente 2}

Producto del tercer embarazo, con edad materna al nacimiento de 39 años, parto hospitalario a las 37 semanas de gestación sin complicaciones, al nacimiento peso de $801 \mathrm{~g}(\mathrm{p}<5)$, talla: $33 \mathrm{~cm}(\mathrm{p}<5)$, PC: $22 \mathrm{~cm}(\mathrm{p}<5)$, distancia intercantal interna de: $1,2 \mathrm{~cm}(\mathrm{p}<5)$, distancia intercantal externa de $4,4 \mathrm{~cm}(\mathrm{p}<5)$, tamaño total de la mano de $3,8 \mathrm{~cm}(\mathrm{p}<5)$, pie: $4,5 \mathrm{~cm}(\mathrm{p}$ $<5$ ), sexo femenino. Al examen físico se encontró microcefalia, fontanela anterior pequeña, fisuras palpebrales inclinadas
Tabla 1. Comparación de las manifestaciones clínicas típicas reportadas por Majewski y Goecke con los pacientes actualmente reportados

\begin{tabular}{|c|c|c|c|c|}
\hline Hallazgos & $\mathbf{n}$ & $\%$ & $\begin{array}{c}\text { Paciente } \\
1\end{array}$ & $\begin{array}{c}\text { Paciente } \\
2\end{array}$ \\
\hline $\begin{array}{l}\text { Retardo mental } \\
\text { moderado a severo }\end{array}$ & $17 / 17$ & 100 & ND & + \\
\hline Microcefalia & $17 / 17$ & 100 & + & + \\
\hline $\begin{array}{l}\text { Puente nasal } \\
\text { prominente }\end{array}$ & $17 / 17$ & 100 & + & + \\
\hline Micrognatia & $17 / 17$ & 100 & + & + \\
\hline $\begin{array}{l}\text { Clinodactilia del } \\
\text { quinto dedo }\end{array}$ & $8 / 8$ & 100 & + & + \\
\hline
\end{tabular}

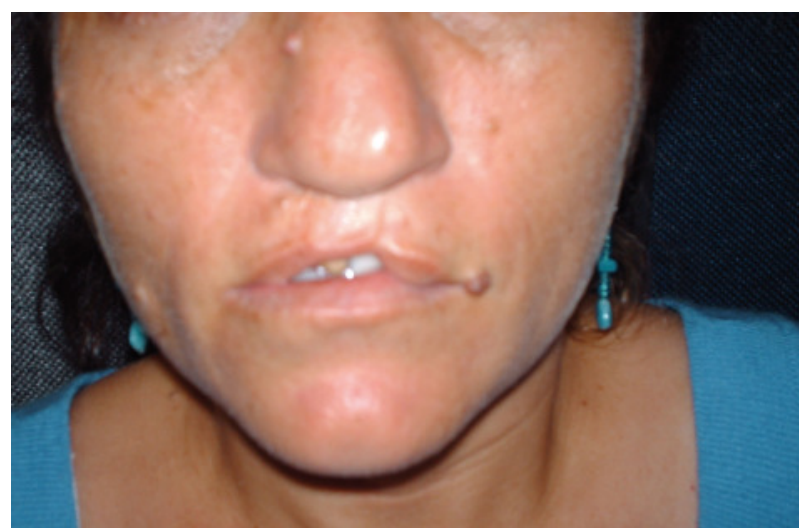

Figura 1. Madre de los pacientes con síndrome de Seckel, nótese cicatriz de labio y paladar hendido bilateral y nariz bulbosa.

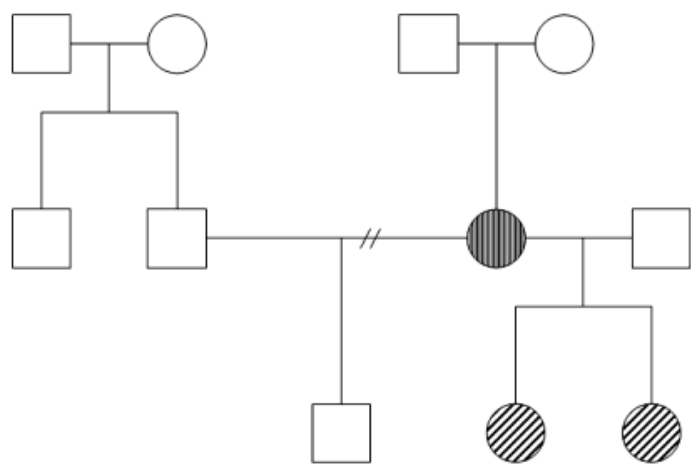

Sindrome de Seckel

\section{Labio y paladar hendido bilateral}

Figura 2. Heredograma de la familia de los pacientes presentados. 


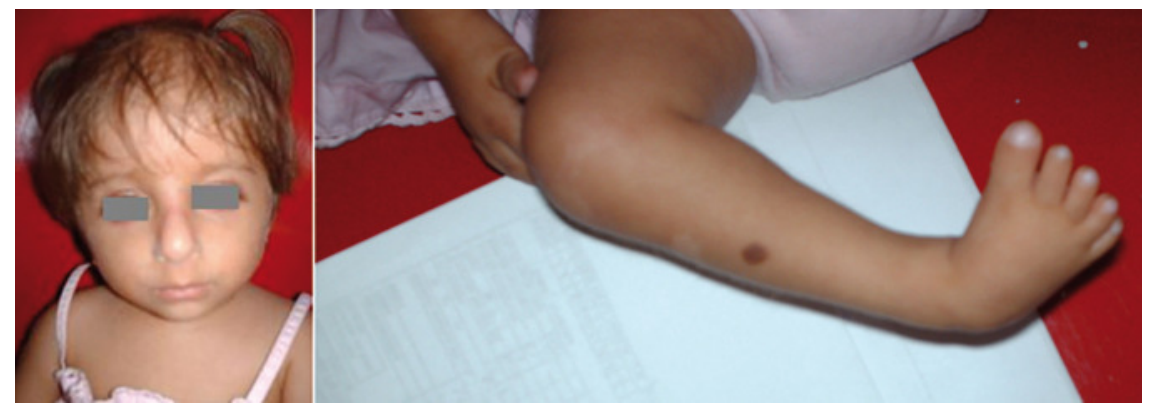

Figura 3. Vista general de paciente 2 con facies típicas de síndrome de Seckel y pie equino varo derecho.

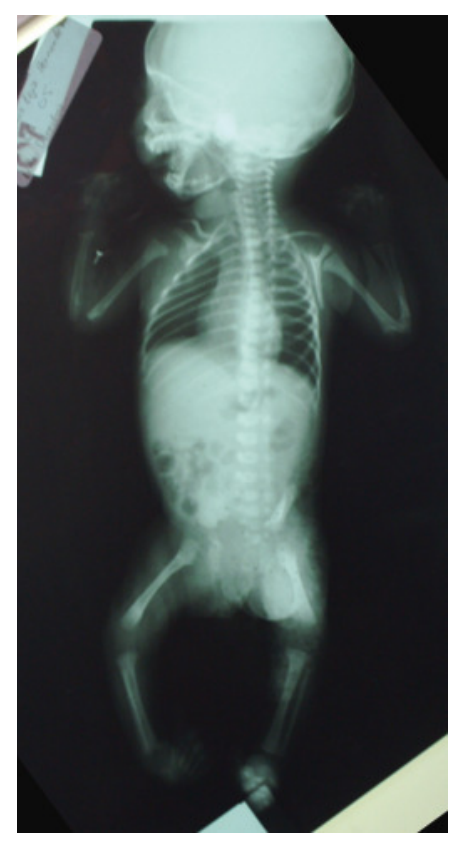

Figura 4. Radiografía de paciente 2 , donde se aprecia 11 costillas y pie equino varo.

hacia abajo, nariz prominente, paladar ojival, micrognatia, frente prominente, clinodactilia bilateral y distensión abdominal (figura 5), además en la radiografía puede observarse una hipoplasia de fémur izquierdo. Se solicitó eco abdominal que evidencia gran quiste meconial, por lo que es llevada a cirugía donde se encontró atresia a nivel del íleon terminal.

Otros exámenes complementarios: ecocardiograma normal, cariotipo bandeo $\mathrm{G}$ con resultado 46 XX.

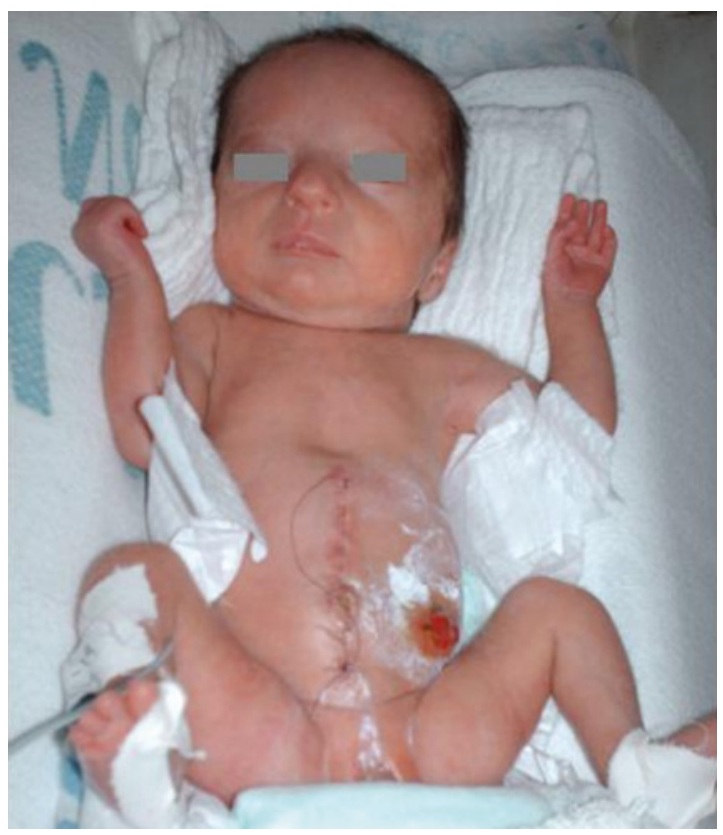

Figura 5. Se evidencia paciente 1 con características fenotípicas de síndrome de Seckel, además se encuentra herida quirúrgica postoperatoria por atresia a nivel del íleon terminal.

\section{Discusión}

El síndrome de Seckel fue descrito inicialmente por Mann y Rusell en $1959^{10}$, y posteriormente con profundidad por Seckel en $1960^{11}$, desde entonces se han reportado cerca de cien $\operatorname{casos}^{2}$, y sólo 30 casos cumplen los criterios propuestos inicialmente por Seckel ${ }^{12}$.

De acuerdo a la revisión realizada por Majewski y Goecke de 17 pacientes con síndrome de Seckel, las características encontradas en el 
Tabla 2. Familias reportadas con casos de Síndrome de Seckel por sexo, autor y año de publicación

\begin{tabular}{|lcccc|}
\hline Autor & n de pacientes & Masculino & Femenino & Consanguinidad familiar \\
\hline Presente reporte & 2 & 0 & 2 & No \\
\hline Black [1961] & 2 & 0 & 2 & No \\
Aarons [1964] & 2 & 1 & 1 & No \\
Harper et al. [1967] & 2 & 1 & 1 & No \\
\hline Sauk et al. [1973] & 4 & 1 & 3 & No \\
Cervenka et al. [1979] & 2 & 2 & 0 & No \\
\hline Krishna et al. [1994] & 3 & 1 & 2 & Sí \\
\hline Shanske [1997] & 3 & 1 & 2 & Sí \\
\hline Faivre et al. [2002] & 2 & 0 & 2 & NR \\
\hline Faivre et al. [2002] & 2 & 0 & 2 & NR \\
\hline Faivre et al. [2002] & 3 & 3 & 0 & NR \\
Faivre et al. [2002] & 2 & 0 & 2 & NR \\
\hline Total de 11 familias & 29 & 10 & 19 & 2 \\
\hline
\end{tabular}

NR: No reportada.

$100 \%$ de los pacientes con este síndrome incluyen retardo en el crecimiento intrauterino con un promedio de peso de $1543 \mathrm{~kg}$ (rango 1,0 a $2055 \mathrm{~kg}$ ), retardo mental moderado a severo, microcefalia, puente nasal prominente, micrognatia, y clinodactilia (tabla 1). Hallazgos fenótipicos que se deben considerar necesarias para realizar el diagnostico de este síndrome ${ }^{1}$ y que fueron encontradas en nuestros pacientes (el retardo mental en el paciente 2, no es posible valorarlo por la edad aunque presenta retardo en el desarrollo sicomotor).

Otros defectos congénitos importantes que permiten distinguir este síndrome, incluyen el puente nasal prominente, frente amplia que contribuyen a la mal llamada expresión "cabeza de pájaro", orejas de implantación baja malformadas y ausencia de lóbulo, ojos grandes, pliegue único, frente inclinada hacia atrás, luxación de cadera, hipoplasia de la región del peroné, incapacidad para la extensión de las rodillas, 11 costillas, y en el sexo masculino criptorquidea. Otras malformaciones ocasionales incluyen anodoncia parcial, talipes, y escoliosis. Sin embargo, estas manifestaciones pueden ser influenciadas por la edad y la etnia ${ }^{13}$. Otros sistemas comprometidos son el osteomuscular, neurológico, hematológico, y ocular $^{14,15}$, pero en la literatura revisada no se encontraron reportes de compromiso del sistema gastrointestinal, siendo este el primer reporte de un caso con síndrome de Seckel asociado a atresia ileal, asociación que podría ser casual.

Las características craneofaciales permiten diferenciar al síndrome de Seckel de otros síndromes caracterizados por baja talla, tales como el síndrome de Dubowitz, síndrome de alcohol fetal, trisomia 18, síndrome de Lange, síndrome de Bloom y síndrome de Fanconi. A diferencia de estos síndromes, el síndrome de Seckel presenta una gran heterogeneidad fenotípica y genotípica, y un número de variantes han sido reportadas ${ }^{2,16}$.

El síndrome de Seckel es un desorden genético de la reparación del DNA y tres tipos han sido propuestos con base a las manifestaciones clínicas y el gen comprometido ${ }^{17}$. En el año 2000 fue mapeado el primer gen para el síndrome de Seckel en el cromosoma 3q22.1q24 (SCKL1) en 2 familias consanguíneas originarias de Pakistán ${ }^{18}$. Otro locus fue mapeado en el cromosoma 18p11.31-q11.2 (SCKL2) en una familia iraquí ${ }^{19}$ y en el 2003 el grupo de Kilinc reporta el tercer locus en el cromosoma 14q21-q22 (SCKL3) en 13 familias Turcas ${ }^{20}$. 


\section{Asesoramiento genético, riesgo de recurrencia y manejo}

El asesoramiento genético se debe enfocar como una enfermedad autosómica recesiva, con un riesgo de recurrencia del $25 \%$ para parejas que hayan tenido un hijo con este síndrome.

El manejo de estos pacientes es sintomático, realizado por un equipo multidisciplinario, acompañado de un soporte psicosocial, la valoración hematológica es necesaria por la asociación con alteraciones hematológicas, un manejo adecuado de la caries por el equipo de odontología es necesario por el incremento en la ocurrencia de esta enfermedad en estos pacientes, todo lo anterior permitirá que estos pacientes y sus familias obtengan una mejor calidad de vida ${ }^{7}$.

\section{Conclusiones}

La presentación de dos o más casos de síndrome de Seckel en una misma familia son pocos frecuentes, en la literatura revisada siendo este el primer reporte. Aunque existe un sobrediagnóstico de este síndrome, la sospecha se debe realizar cuando se presente un niño con una talla baja severa, retardo mental, microcefalia severa, frente prominente, ojos grandes, protrusión de la nariz, cara pequeña y micrognatia.

\section{Referencias}

1.- Majewski F, Goecke T: Studies of microcephalic primordial dwarfism. I: Approach to a delineation of the Seckel syndrome. Am J Med Genet 1982; 12: 7-21.

2.- Faivre L, Le Merrer M, Lyonnet $S$, et al: Clinical and genetic heterogeneity of Seckel syndrome. Am J Med Genet 2002; 112: 369-83.

3.- Black J: Low birth weight dwarfism. Arch Dis Child 1961; 36: 633-44.

4.- Aarons PH: Vogelkopdwergen. Maandschr Kindergeneesk 1964; 32: 384-94.

5.- Harper RG, Orti E, Baker RK: Bird headed dwarfs (Seckel's syndrome). J Pediatr 1967; 70: 799-804.

6.- SaukJJ, Litt R, Espiritu CE, Delaney IR: Familial birdheaded dwarfism (Seckel's syndrome). J Med Genet
1973; 10: 196-8.

7.- Cervenka J, Tsuchiya H, Ishiki T, Suziki M, Mori H: Seckel's dwarfism: Analysis of chromosome breakage and sister chromatid exchanges. Am J Dis Child 1979; 133: 555-6.

8.- Krishna AG, Scrimgeour EM, Zawawi TH: Seckel syndrome in a Yemeni family in Saudi Arabia. Am J Med Genet 1994; 51: 224-7.

9.- Shanske A, Caride DG, Menasse-Palmer L, Bogdanow A, Marion $R W$ : Central Nervous System Anomalies in Seckel Syndrome: Report of a New Family and Review of the Literatura American Journal of Medical Genetics 1997; 70: 155-8.

10.- Mann TP, Rusell A: Study of a microcephalic midget of extreme type. Proc R Soc Med 1959; 52: 1024.

11.- Seckel HPG: Bird-Headed Dwarfs: Studies in Developmental Anthropology Including Human Proportions. Springfield: Charles C. Thomas, 1960.

12.- Hennekam RCM, Krantz IA, Allanson JE: Syndromes of the Head and Neck, five edition. New York: Oxford University Press 2010; pp 457-62.

13.- Buebel MS, Salinas CF, Pai GS, Macpherson RI, Creer MK, Pérez A: A New Seckel-Like Syndrome of Primordial Dwarfism. Am J Med Genet 1996; 64: 447-52.

14.- Thompson E, Pembrey M: Seckel syndrome: an overdiagnosed syndrome. J Med Genet 1985; 22: 192-201.

15.- Reddy S, Starr C: Seckel syndrome and spontaneously dislocated lenses. J Cataract Refract Surg 2007; 33: 9102.

16.- De Coster PJ, Verbeeck RM, Holthaus V, Martens LC, Vral A: Seckel syndrome associated with oligodontia, microdontia, enamel hypoplasia, delayed eruption, and dentin dysmineralization: a new variant?. J Oral Pathol Med 2006; 35: 639-41.

17.- Rayburg M, Davies SM, Mehta PA, Crockett M, Jodele $S$ : Successful reduced-intensity bone marrow transplantation in a patient with bone marrow failure associated with Seckel syndrome. British Journal of Haematology 2008; 142: 668-80.

18.- Goodship J, Gill H, Carter J, Jackson A, Splitt M, Wright M: Autozygosity mapping of a Seckel syndrome locus to chromosome 3q22.1-q24. Am J Hum Genet 2000; 67: 498-503.

19.- Borglum AD, Balslev T, Haagerup A, et al: A new locus for Seckel syndrome on chromosome 18p11.31-q11.2. Eur J Hum Genet 2001; 9: 753-7.

20.- Kilinc MO, Ninis $V N$, Ugur SA, et al: Is the novel SCKL3 at $14 \mathrm{q} 23$ the predominant Seckel locus?. Eur J Hum Genet 2003; 11: 851-7. 